\section{Incidence, Risk Factors, and Outcome of Acute Kidney Injury in Neurocritical Care}

Journal of Intensive Care Medicine 2020, Vol. 35(4) 338-346

(C) The Author(s) 2018

Article reuse guidelines:

sagepub.com/journals-permissions DOI: $10.1177 / 0885066617748596$

journals.sagepub.com/home/jic

(S)AGE

\author{
Stefan Büttner, MD', Andrea Stadler, MD', Christoph Mayer, MD², \\ Sammy Patyna, MD', Christoph Betz, MD', Christian Senft, MD³, \\ Helmut Geiger, MD', Oliver Jung, MD', and Fabian Finkelmeier, $M D^{3,4}$
}

\begin{abstract}
Purpose: Acute kidney injury (AKI) is a severe complication in medical and surgical intensive care units accounting for a high morbidity and mortality. Incidence, risk factors, and prognostic impact of this deleterious condition are well established in this setting. Data concerning the neurocritically ill patients is scarce. Therefore, aim of this study was to determine the incidence of AKI and elucidate risk factors in this special population. Methods: Patients admitted to a specialized neurocritical care unit between 2005 and 201 I with a length of stay above 48 hours were analyzed retrospectively for incidence, cause, and outcome of AKI (AKI Network-stage $\geq 2$ ). Results: The study population comprised $68 \mathrm{I}$ neurocritically ill patients from a mixed neurosurgical and neurological intensive care unit. The prevalence of chronic kidney disease (CKD) was 8.4\% (57/68I). Overall incidence of AKI was I I.6\% with 36 (45.6\%) patients developing dialysis-requiring AKI. Sepsis was the main cause of AKI in nearly $50 \%$ of patients. Acute kidney injury and renal replacement therapy are independent predictors of worse outcome (hazard ratio [HR]: 3.704; 95\% confidence interval [Cl]: I.867-7.350; $P<.001$; and HR: 2.848; $\mathrm{Cl}: 1.30 \mathrm{I}-6.325 ; P=.009)$. Chronic kidney disease was the strongest independent risk factor (odds ratio: 12.473; Cl: 5.944-26.172; $P<.001$ ), whereas surgical intervention or contrast agents were not associated with AKI. Conclusions: Acute kidney injury in neurocritical care has a high incidence and is a crucial risk factor for mortality independently of the underlying neurocritical condition. Sepsis is the main cause of AKI in this setting. Therefore, careful prevention of infectious complications and considering CKD in treatment decisions may lower the incidence of $\mathrm{AKI}$ and hereby improve outcome in neurocritical care.
\end{abstract}

\title{
Keywords
}

acute kidney injury, neurocritical care, outcome, complications

\section{Introduction}

Non-neurologic complications in neurocritical care are increasingly recognized as independent predictors of poor outcome in this subset of critically ill patients. ${ }^{1}$ Acute kidney injury (AKI) is a common and severe complication in medical and surgical intensive care units (ICUs) accounting for high morbidity and mortality in these patients. ${ }^{2}$ Large studies have shown an incidence of $20 \%$ to $67 \%$ depending on the definition of AKI and the underlying cause of admission to the ICU with the lowest incidence in elective surgical patients and the highest incidence in patients with sepsis. ${ }^{2-6}$ Consequently, AKI requiring renal replacement therapy (RRT) is an important risk factor for mortality, with reported mortality rates up to $50 \%$ to $70 \%$ in these patients. $5,7,8$

Furthermore, AKI is associated with increasing costs, ${ }^{9}$ prolonged ICU and hospital stay, ${ }^{10}$ and an elevated risk of chronic kidney disease (CKD) including end-stage renal disease. . $^{811,12}$ Incidence, risk factors, and prognostic impact of this deleterious condition are well established in the setting of medical and surgical ICU. However, to date, only few data about the incidence and impact of AKI in the neurocritically ill patient are published, mostly concerning individual populations like patients after stroke, ${ }^{13-15}$ traumatic brain injury, ${ }^{16,17}$ and intracerebral ${ }^{18}$ or subarachnoid hemorrhage. ${ }^{19}$ Nevertheless, those

\footnotetext{
' Medical Clinic III, Department of Nephrology, University Hospital Frankfurt, Goethe University, Frankfurt am Main, Germany

${ }^{2}$ Department of Neurology, University Hospital Frankfurt, Goethe University, Frankfurt am Main, Germany

${ }^{3}$ Department of Neurosurgery, University Hospital Frankfurt, Goethe University, Frankfurt am Main, Germany

${ }^{4}$ Medical Clinic I, Gastroenterology, Hepatology, University Hospital Frankfurt, Goethe University, Frankfurt am Main, Germany
}

Received July 12, 2017. Received revised November 23, 2017. Accepted November 27, 2017.

\section{Corresponding Author:}

Stefan Büttner, Medical Clinic III, Department of Nephrology, University Hospital Frankfurt, Goethe University, Theodor-Stern-Kai 7, D-60590 Frankfurt/Main, Germany.

Email: stefan.buettner@kgu.de 
patients are often of younger age and show different spectra of underlying disease as well as reduced numbers of comorbidities than their counterparts in medical or surgical ICU. Diagnostic and therapeutic procedures in neurocritical care may exhibit a different risk profile regarding the development of AKI.

The aim of this study was to evaluate incidence and outcome of AKI in a large and unselected cohort of neurocritically ill patients in a specialized neurocritical care unit (NCCU). In addition, we sought to elucidate risk factors for the development of AKI and describe the impact of AKI on survival in this special subpopulation of critically ill patients.

\section{Materials and Methods}

\section{Selection of Patients}

All patients admitted to the NCCU of the University Hospital Frankfurt, a tertiary care clinic in a large metropolitan area, between January 2005 and December 2011, were included in this retrospective cohort study. The NCCU is a specialized ward with 16 intensive care beds shared for neurological and neurosurgical patients. The team consists of neurosurgeons, neurologists, anesthetists, and a specialized intensive care nursing staff. A consultative support is organized in cooperation with the other clinics of the hospital to address non-neurological problems (eg, the nephrologist on call in case of AKI). The study was performed in accordance with the 1975 Declaration of Helsinki, and the study was approved by the institutional review board of the University Hospital Frankfurt (Geschäfts-Nr. 59/12). Patients admitted for less than 48 hours and patients who were diagnosed brain dead or had "allow natural death" orders at the time of admission were excluded. Also excluded were patients with withdrawal of care (advance health-care directive or at request by the family) before or at the onset of AKI and individuals undergoing chronic RRT (either hemodialysis or peritoneal dialysis) prior to admission. Demographic, clinical, laboratory, and treatment parameters were retrieved from the electronic medical case files for every patient. To assess severity of critical illness, the Simplified Acute Physiology Score (SAPS II) was used. ${ }^{20}$

\section{Definition of AKI}

Baseline kidney function was estimated at admission based on the first serum creatinine value and the corresponding estimated glomerular filtration rate (eGFR) using the 4-variable modification of diet in renal disease formula or, if available, from previous hospital stays (at least 3 months prior to admission). Chronic kidney disease was defined if eGFR was below $90 \mathrm{~mL} / \mathrm{min} / 1.73 \mathrm{~m} 2$ or if a history of CKD was known. Acute kidney injury was defined according to the Acute Kidney Injury Network (AKIN) staging system. ${ }^{21}$ For the purpose of this study, patients were classified as having AKI if reached AKIN-stage 2 (serum creatinine increase $>2$ - to 3 -fold increase from baseline) or 3 (serum creatinine increase above 3 -fold or serum creatinine $>4 \mathrm{mg} / \mathrm{dL}$ with an acute increase $\geq 0.5 \mathrm{mg} / \mathrm{dL}$ ). Diagnosis and cause of AKI was confirmed in every single case by retrospective analysis of the medical case files by 2 different nephrologists independently. Causes were attributed to sepsis, nephrotoxic, pre- or postrenal, postsurgery, or others/unknown. To assess and elucidate risk factors for AKI, the cohort of patients was divided in 2 groups, patients without AKI as a control group and patients with AKIN of 2 or higher. In a second analysis, only the identified patients with AKI were divided in 2 groups. The first group comprised patients in which kidney function could be stabilized with conventional treatment alone. The second group includes patients with AKI requiring RRT. Patients were followed up to death or up to 90 days post-ICU admission.

\section{Definitions}

Admission diagnoses were categorized as neurologic autoimmune disease, intracranial hemorrhage (including intracerebral, extracerebral, and subarachnoid hemorrhages), infection of the central nervous system, ischemic stroke, tumor disease, and others. Classified comorbidities were arterial hypertension, diabetes, coronary artery disease (CAD), peripheral arterial disease (PAD), any known cerebrovascular disease (CVD), and CKD. The use of contrast agent (computed tomography or angiography) during the course of treatment, antimicrobial therapy (excluding specific nephrotoxic medications as given below), and the rate of confirmed infections for every single patient was recorded. Diagnosis of sepsis was based on the sepsis definition of the American College of Chest Physicians/Society of Critical Care Medicine Consensus Conference Committee. ${ }^{22}$ Diagnosis of ventilator-associated pneumonia was based on the guidelines of the American Thoracic Society. ${ }^{23}$ The variable nephrotoxic agents included vancomycin, non-steroidal anti-inflammatory drugs, ganciclovir, acyclovir, tobramycin, streptomycin, gentamycin, amikacin, cyclosporine, and amphotericin in any single or combined use.

\section{Statistical Analysis}

Continuous variables are shown as median and interquartile range (IQR), and categorical variables are reported as frequencies and percentages. Differences between patient cohorts were determined using the Fishers exact test or Fisher-FreemanHalton exact test for categorical variables; for quantitative variables, we used the Mann-Whitney $U$ test or Kruskal-Wallis test. Risk factors for AKI were determined using a uni- and multivariate logistic regression model. For assessment of survival factors, we used a uni- and multivariate Cox-regression model. All $P$ values reported are 2-sided. Statistical significance was assumed when the $P$ value was $<.05$. Statistical analyses were performed using SPSS 22 (IBM, Armonk, New York) and Prism 5 (GraphPad Software Inc, San Diego, California).

\section{Results \\ Patient Characteristics}

The study population comprised 681 patients with a median age of 62.5 (IQR, 49-73) years with 377 (55.4\%) males (for 
Table I. Demographic Data and Clinical Characteristics of the Control Cohort and AKI Patients. ${ }^{a}$

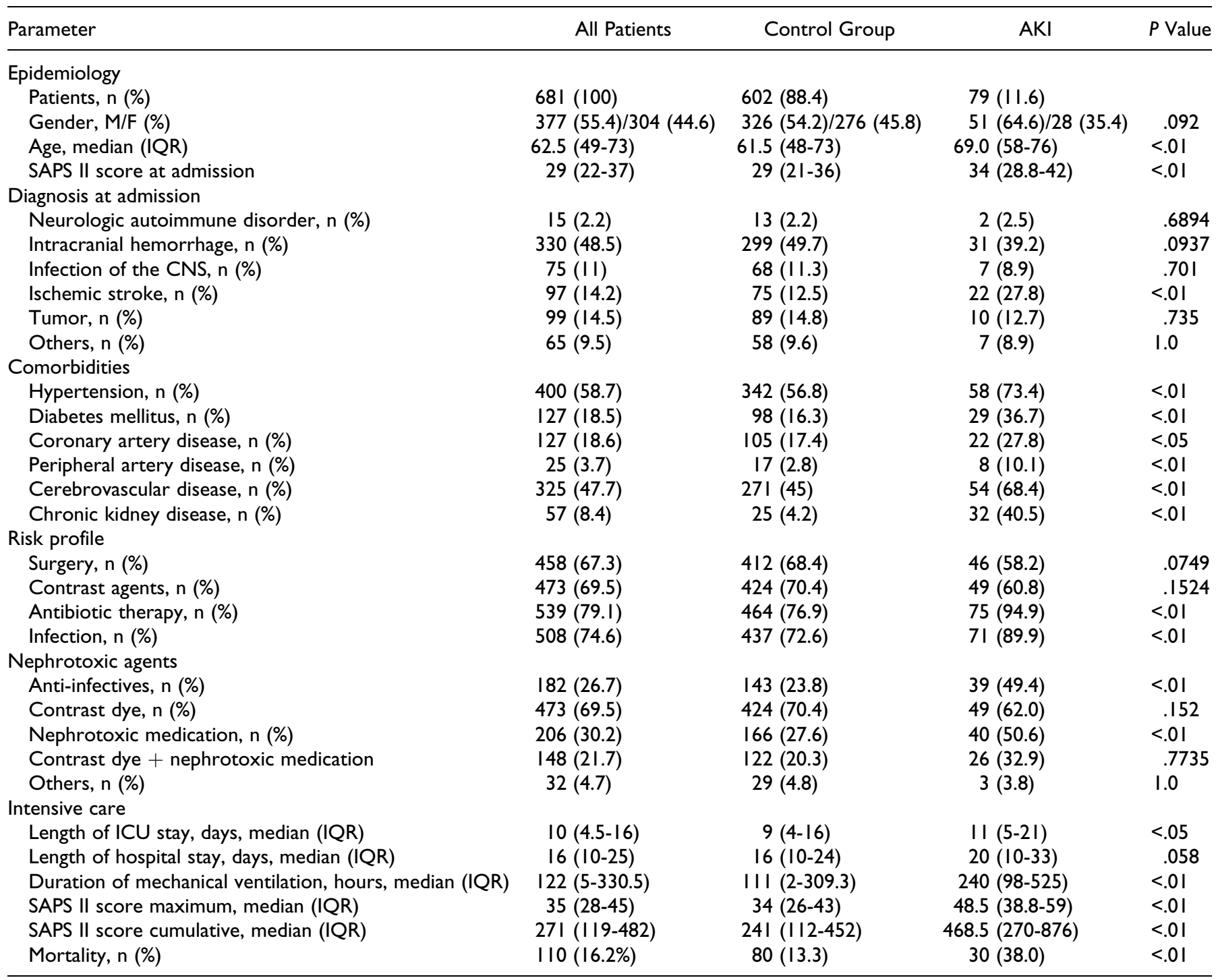

Abbreviations: AKI, acute kidney injury; CNS, central nervous system; F, female; ICU, intensive care unit; IQR, interquartile range; M, male; SAPS, Simplified Acute Physiology Score.

${ }^{a}$ Variables are expressed as median and IQR or as numbers and proportions, as appropriate. All $P$ values reported are 2 -sided. Statistical significance was defined as $P \leq .05$.

detailed data see Table 1). Median ICU stay was 10 (4.5-16) days, with a median duration of mechanical ventilation of 122 (5-330.5) hours and a median SAPS II score of 29 (22-37) at the time point of admission. Reasons for admittance were mainly due to intracranial hemorrhage (48.5\%), followed by intracerebral neoplasms $(14.5 \%)$, ischemic stroke $(14.2 \%)$, and infections of the central nervous system (11\%). Following hypertension (58.7\%), CVD (47.7\%) was the second most common comorbidity. Diabetes mellitus (18.5\%) and CAD $(18.6 \%)$ were equally frequent and 57 patients $(8.4 \%)$ had preexisting CKD. In all, $458(67.3 \%)$ patients underwent surgery right before or during ICU stay, 473 (69.5\%) received contrast agents, and 508 patients were diagnosed with an infection $(74.6 \%)$.

\section{Patients With AKI}

Throughout the study period, 79 patients developed AKI (AKIN-stage $\geq 2$ ), accounting for an incidence of $11.6 \%$. As depicted in Table 1, this group included 51 (64.6\%) males and $28(35.4 \%)$ females with a median age of 69 (58-76) years, which was significantly older than the control group $(P<$ $.001)$. Of total, 27 (34.2\%) patients were classified as AKINstage 2 and $52(65.8 \%)$ patients as AKIN-stage 3. Admission diagnoses did only differ significantly in patients with ischemic stroke with $27.8 \%(\mathrm{n}=22)$ in the AKI group and $12.5 \%(\mathrm{n}=$ $75)$ in the control group $(P<.01)$. Patients in the AKI group significantly more often had comorbidities like hypertension (73.3\% vs $56.8 \% ; P<.01)$, diabetes $(36.7 \%$ vs $16.3 \% ; P<.01)$, 
Table 2. Demographic Data and Clinical Characteristics of Patients With AKI and Renal Replacement Therapy. ${ }^{\mathrm{a}}$

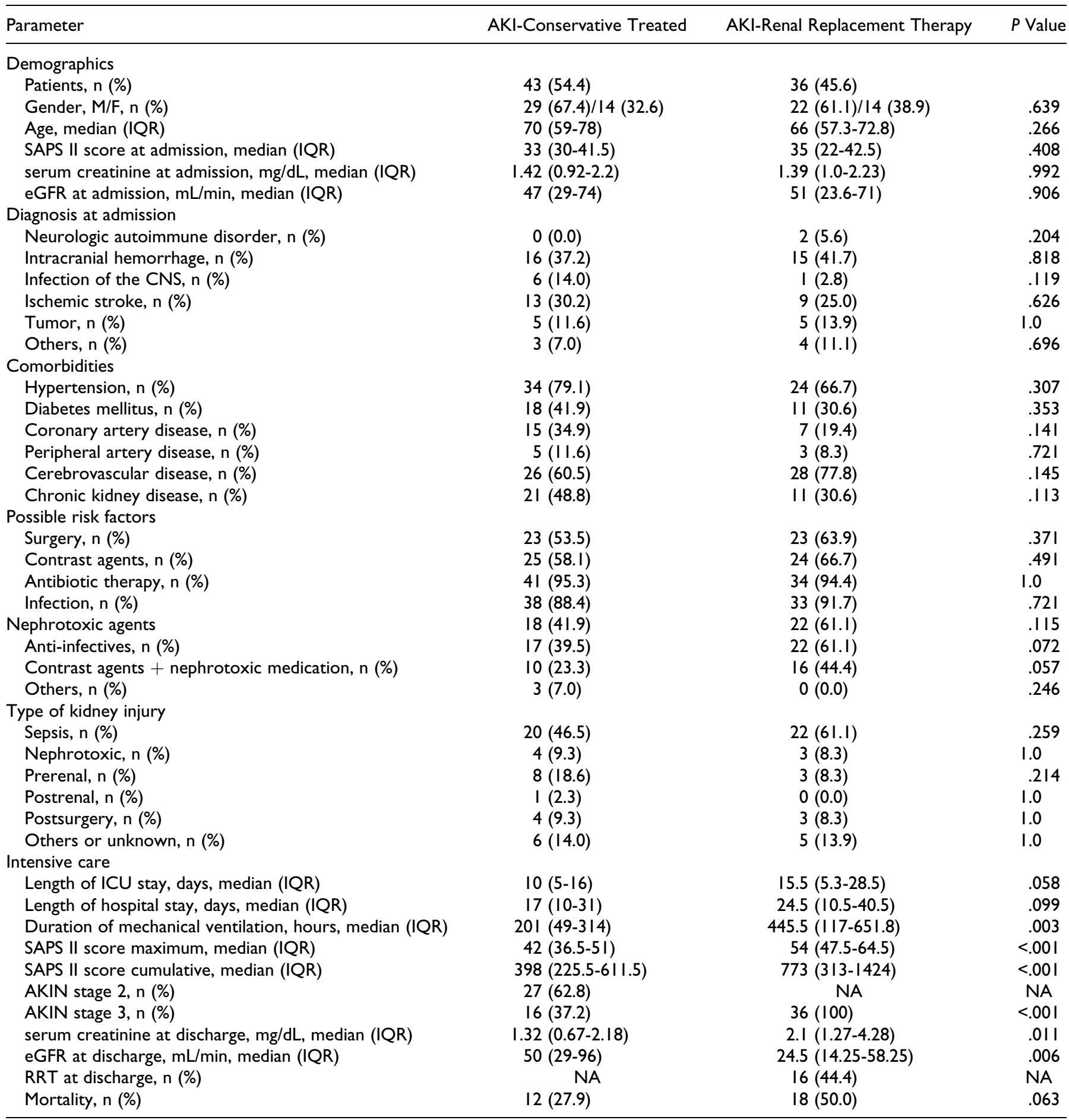

Abbreviations: AKI, acute kidney injury; AKIN, Acute Kidney Injury Network; CNS, central nervous system; eGFR, estimated glomerular filtration rate; F, female; ICU, intensive care unit; IQR, interquartile range; M, male; NA, not applicable; RRT, renal replacement therapy; SAPS, Simplified Acute Physiology Score. ${ }^{a}$ Variables are expressed as median and IQR or as numbers and proportions, as appropriate. All $P$ values reported are 2 -sided. Statistical significance was defined as $P \leq .05$.

CAD $(27.8 \%$ vs $17.4 \% ; P<.05)$, PAD $(10.1 \%$ vs $2.8 \%$; $P<.01)$, CVD (68.4\% vs $45 \% ; P<.01)$, and CKD $(40.5 \%$ vs $4.2 \% ; P<.01)$. Regarding possible risk factors, significantly more patients in the AKI group were diagnosed with infection $(89.9 \%, \mathrm{n}=71 ; P<.01)$, underwent antibiotic therapy $(94.9 \%, \mathrm{n}=75 ; P<.01)$, and received nephrotoxic agents (mainly anti-infectives; $50.6 \%$, $\mathrm{n}=40 ; P<.01)$. 
Table 3. Univariate and Multivariate Analyses of Risk Factor Significantly Associated With AKI. ${ }^{a}$

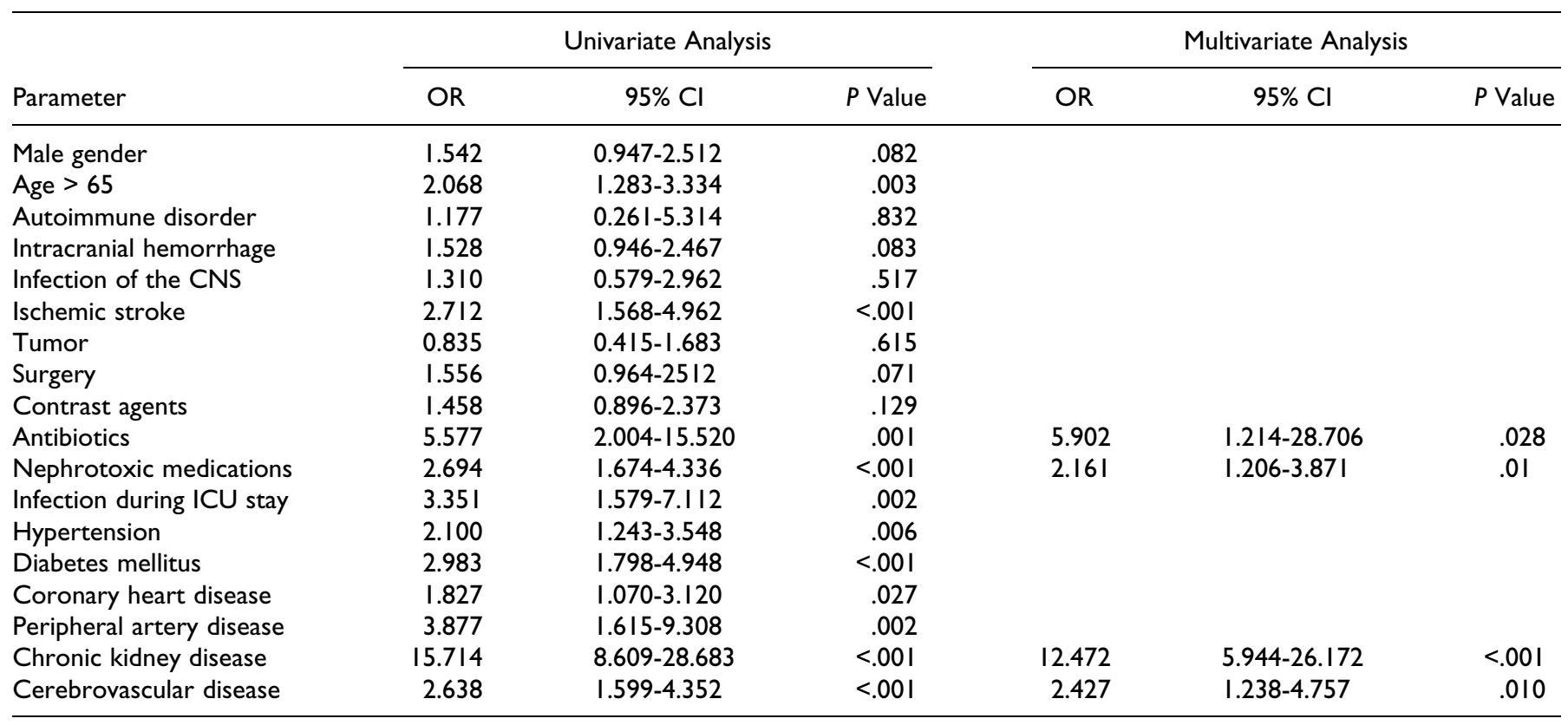

Abbreviations: AKI, Acute Kidney Injury; $\mathrm{Cl}$, confidence interval; CNS, central nervous system; ICU, intensive care unit; OR, odds ratio.

${ }^{a}$ All $P$ values reported are 2 -sided, and all confidence intervals are $95 \%$ intervals. Statistical-significance was defined as $P \leq .05$.

Sepsis was the main cause of AKI in 42 patients (53.2\%), with $69 \%$ of these cases due to pneumonia. The second most cause was prerenal AKI in $11(13.6 \%)$ patients, followed by postoperative AKI in 7 (8.9\%) patients, nephrotoxic in 7 (8.9\%) patients, and postrenal AKI in $1(1.3 \%)$ patient. Other causes were rhabdomyolysis $(\mathrm{n}=1)$, hepatorenal syndrome $(\mathrm{n}=1)$, or undefined causes in 11 patients $(13.9 \%)$.

Patients with AKI had a significant longer median ICU stay with 11 (5-21) days versus 9 (4-16) days in the control group $(P<.05)$, a longer duration of mechanical ventilation with 240 (98-525) hours versus $111(2-309.3)$ hours $(P<.01)$. The SAPS II score was higher at admission with 34 (28.8-42) versus 29 $(21-36 ; P<.001)$ and showed an increased maximum during stay with $48.5(38.8-59)$ versus $34(26-43 ; P<.01)$. However, length of hospital stay differed not significantly between the groups with $20(10-33)$ days in the AKIN group versus $16(2-125)$ days in the control group $(P=.058)$.

\section{Patients With the Need for RRT}

Conservative treatment was sufficient in 43 (54.4\%) of all patients with AKI. Thirty-six (45.6\%) patients developed dialysis-dependent AKI, accounting for an overall incidence of dialysis-depending AKI of 5.3\%. Comparing both groups (for detailed data see Table 2), there was no difference regarding admission diagnosis, comorbidities, or possible risk factors (surgery, contrast agent, antibiotic therapy, and infection). However, the number of patients treated with nephrotoxic anti-infectives or the combination with contrast agents was slightly higher in the RRT group without reaching significance. Patients with the need for RRT had a longer stay on ICU and had a significantly longer duration of mechanical ventilation.
Neither the SAPS II score nor kidney function did differ significantly at admission. After discharge from ICU, 16 (44.4\%) of 36 patients were still on RRT; however 90 days after ICU admission, only 2 (5.6\%) patients remained on RRT.

\section{Risk Factors for AKI}

For the identification of risk factors associated with the development of AKI, we conducted a binary logistic regression (for detailed data see Table 3). In a univariate analysis, we found an age above 65 years, several comorbidities, namely hypertension, diabetes mellitus, $\mathrm{CAD}, \mathrm{PAD}, \mathrm{CVD}$, and $\mathrm{CKD}$ as significant risk factors. Except for ischemic stroke, no underlying disease was significantly associated with AKI. Regarding the risk profile antibiotic therapy, nephrotoxic medication and infection were associated with the development AKI. In multivariate analysis, independent risk factors for AKI were CKD (odds ratio [OR]: 12.472; 95\% confidence interval [CI]: 5.944$26.172 ; P<.0001$ ), the use of antibiotics (OR: $5.902 ; 95 \% \mathrm{CI}$ : 1.214-28.706, $P=.028$ ), and the use of nephrotoxic medication (OR: $2.161 ; 95 \%$ CI: 1.206-3.871; $P=.01$ ). Surgical intervention or the administration of contrast agents was not associated with AKI. Interestingly, CVD was an independent risk factor for the development of AKI (OR: 2.427; 95\% CI: $1.238-4.757 ; P=.01)$.

\section{Outcome and Survival Analysis}

Overall mortality was $16.2 \%(\mathrm{n}=110)$ during the 90 -day follow-up (94 [13.8\%] patients died within 28 days after admission), with death occurring after a median of 9.5 (full range: 2-90) days. Compared to patients without AKI, those with any 

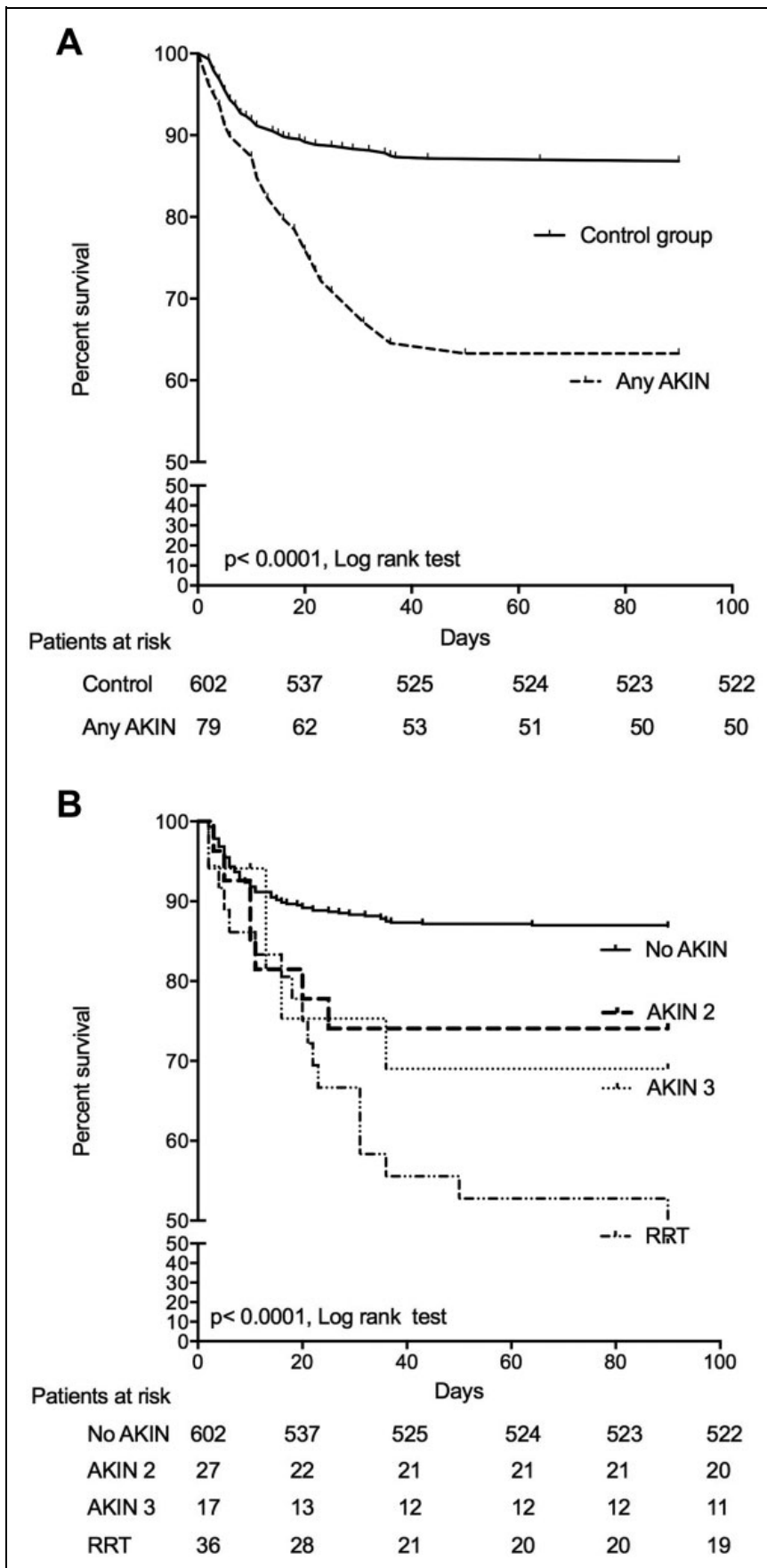

Figure I. Overall survival according to AKI and different stages of AKIN. Overall survival of patients with and without acute kidney injury (A) as well as survival of patients with AKIN stages 2, 3, or 3 with renal replacement therapy (B) assessed by a univariate Cox regression models. AKIN indicates Acute Kidney Injury Network.

stage of AKI were significantly more likely to die (38\% vs $13.3 \% ; P<.01$; Figure $1 \mathrm{~A}$ ). With increasing severity of AKI, mortality increased dramatically (no AKIN: $13.3 \%$; AKIN 2: 25.9\%; AKIN 3 no RRT: $31.3 \%$, AKIN 3 with RRT: 50\%; Figure 1B). Survivors were significantly younger, had significantly lower rates of CAD or PAD and had a shorter time on mechanical ventilation and a lower SAPS II score at admission.
Furthermore, the rate of patients with AKI in the nonsurvivor group was higher $(27.3 \%$ vs $8.6 \% ; P<.01)$. Interestingly the number of patients with diagnosed infection or the use of antibiotics was significantly higher in the survivor group (for detailed information see Table 4).

As shown in Table 5, univariate analysis revealed several factors significantly associated with mortality, whereas in multivariate analysis, CKD (2.290; 95\% CI: 1.097-4.781; $P=.027)$, AKI $(3.704 ; 95 \%$ CI: $1.867-7.350 ; P<.001)$, the need for RRT (2.848; 95\% CI: 1.301-6.325; $P=.009)$, and the use of antibiotics (8.117; 95\% CI: 2.090-31.523; $P=.002)$ were independent risk factors for death.

\section{Discussion}

Neurocritical illness is often associated with a poor prognosis. Specialized neurocritical care is one of the newer disciplines of intensive care medicine and evolved from neurosurgical postoperative monitoring and treatment. The advent of neurocritical care led to a dramatic decrease in mortality and improved prognosis of the neurocritically ill patient. ${ }^{24,25}$ Thus, prognosis of this patient group is increasingly determined by nonneurological complications, ${ }^{1,26}$ rather than the underlying neurologic or neurosurgical diseases. Among these non-neurologic complications, AKI is an independent risk factor for mortality after traumatic brain damage, ${ }^{17}$ ischemic stroke, ${ }^{13}$ and subarachnoid hemorrhage. ${ }^{19}$ Nevertheless, this is the first study examining an unselected, general population of neurocritical ill patients.

In this retrospective single-center analysis of 681 unselected neurocritically ill patients, cumulative incidence of AKI was $11.6 \%$. This goes in line with studies reporting an incidence of AKI after stroke of $14.5 \%{ }^{13}$ or $9.2 \%$ after traumatic brain injury. ${ }^{27}$ However, depending on the study population and the definition of AKI, incidence of AKI in critical care reaches up to $70 \%$. ${ }^{2,8,11,28-30}$ Concerning the incidence of dialysis dependent AKI, we found an incidence of $5.3 \%$, which met the reported $3 \%$ to $12 \%$.

The cause of AKI in medical or surgical critically ill patients is often multifactorial, with sepsis being one of the leading causes in almost $50 \%$ of cases. ${ }^{31}$ Studies showed that about $60 \%$ of patients with sepsis develop AKI and that AKI adversely affect clinical outcome with increasing AKI severity. ${ }^{4,31,32}$ Interestingly, in our cohort, sepsis was the main cause of AKI too. Sepsis promoted AKI in more than half of the cases and the focus was mainly pneumonia, often ventilatorassociated pneumonia. The incidence of AKI during an episode of ventilator-associated pneumonia (VAP) is high (up to 38\%) with multidrug resistant pathogens, sepsis, and disease severity at admission being independent risk factors. ${ }^{33}$ Otherwise, AKI is an acute systemic disease with serious distant organ effects inter alia leading to pulmonary sequelae (eg pulmonary edema), which is associated with a significant higher rate of mechanical ventilation. ${ }^{7,26}$ In our cohort, AKI is associated with a significant longer need of mechanical ventilation, and the use of nephrotoxic medication and antibiotics increased the 
Table 4. Demographic Data and Clinical Characteristics of Survivors and Nonsurvivors. ${ }^{a}$

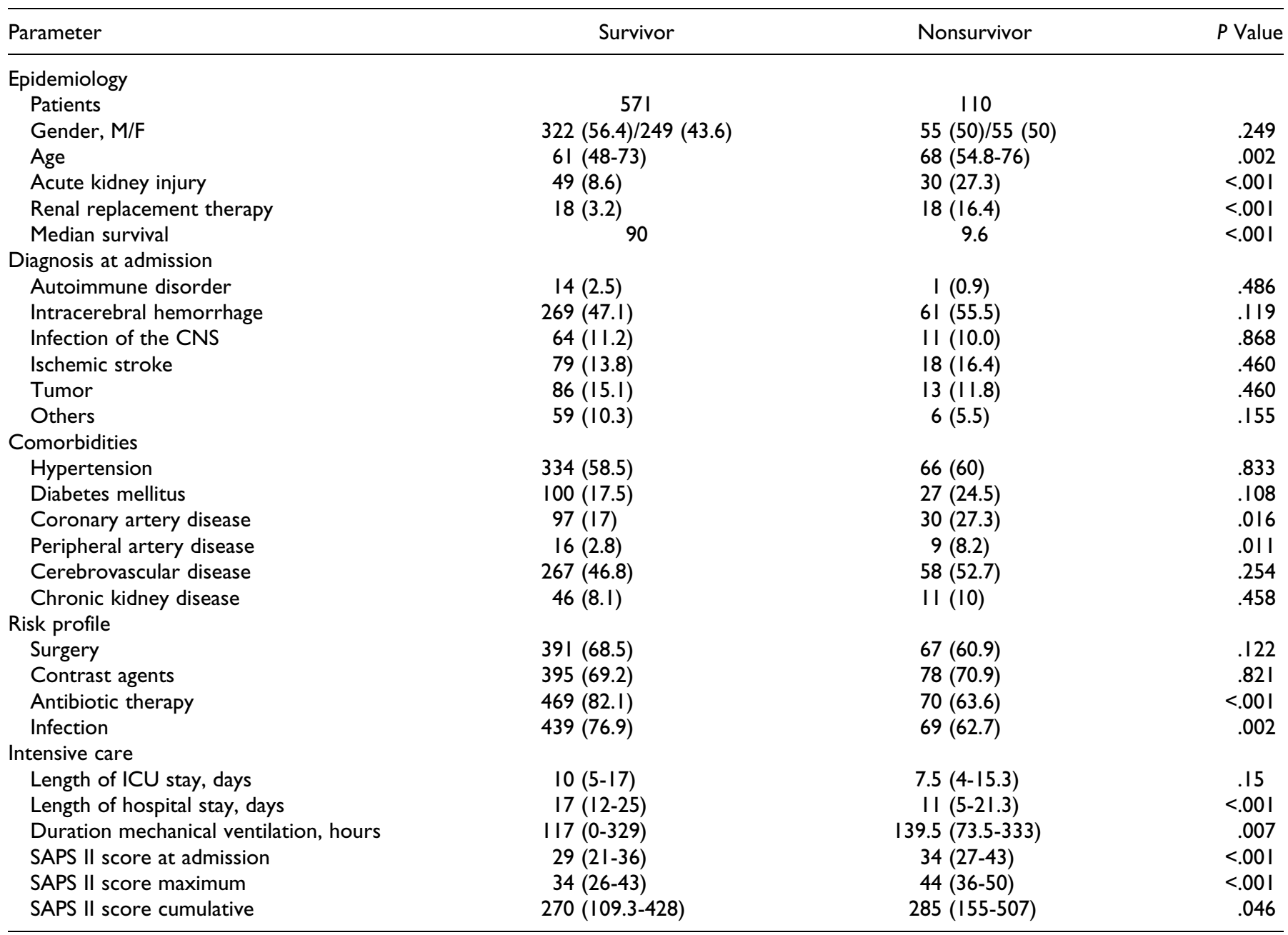

Abbreviations: CNS, central nervous system; F, female; ICU, intensive care unit; M, male; SAPS, Simplified Acute Physiology Score; SD, standard deviation. ${ }^{a}$ Variables are expressed as median and range, mean (SD) or as proportions as appropriate. All $P$ values reported are 2-sided. Statistical significance was defined as $P \leq .05$.

risk for AKI. Strategies to prevent infectious complications like VAP in neurocritically ill patients with prolonged time of mechanical ventilation and early detection of patients at risk are pivotal and may reduce the risk of AKI and related distant organ damage.

In the aforementioned study by Covic et al, independent predictors of AKI were age, GFR, ischemic heart disease, chronic heart failure, and type of stroke. ${ }^{13}$ Patients developing AKI after traumatic brain injury were older, ${ }^{27}$ had lower Glasgow coma scale scores, ${ }^{16,27}$ higher severity of illness scores, ${ }^{27}$ and higher serum creatinine and blood urea nitrogen..$^{16,17,27}$ In our univariate analysis, we could show that classical risk factors like hypertension, diabetes, and CKD are associated with AKI. However, after multivariate analysis, CKD was the strongest independent risk factor for AKI underscoring the need for close monitoring of patients with impaired renal function. Especially dosage adjustments of potential nephrotoxic medications play a critical role in the care of patients with known CKD. Additionally, as shown in multivariate analysis, CVD was an independent risk factor for AKI in our study. Traditional risk factors for cardiovascular and, in particular, CVD, such as advanced age, arterial hypertension, and diabetes mellitus, are also strongly associated with CKD. Patients with CVD, including stroke, therefore represent a vulnerable population with high risk of CKD prone to acute deterioration of kidney function. The fact that we found that no association of AKI with other underlying diseases in our cohort underscores the importance and correlation of CVD with CKD.

Acute kidney injury is an independent risk factor for an unfavorable outcome in patients after ischemic stroke, ${ }^{13-36}$ subarachnoid hemorrhage, ${ }^{19}$ and traumatic brain injury. ${ }^{16,27}$. Moreover, with increasing severity of AKI, mortality increased dramatically in our population, with the highest mortality in patients with need for RRT.

Interestingly, we could not show any association of contrast agents and AKI in our cohort. However, this goes in line with several studies showing a very low incidence of contrastinduced AKI (CI-AKI), showing an incidence of CI-AKI in 
Table 5. Univariate and Multivariate Analyses of Parameters Associated With Survival. ${ }^{\mathrm{a}}$

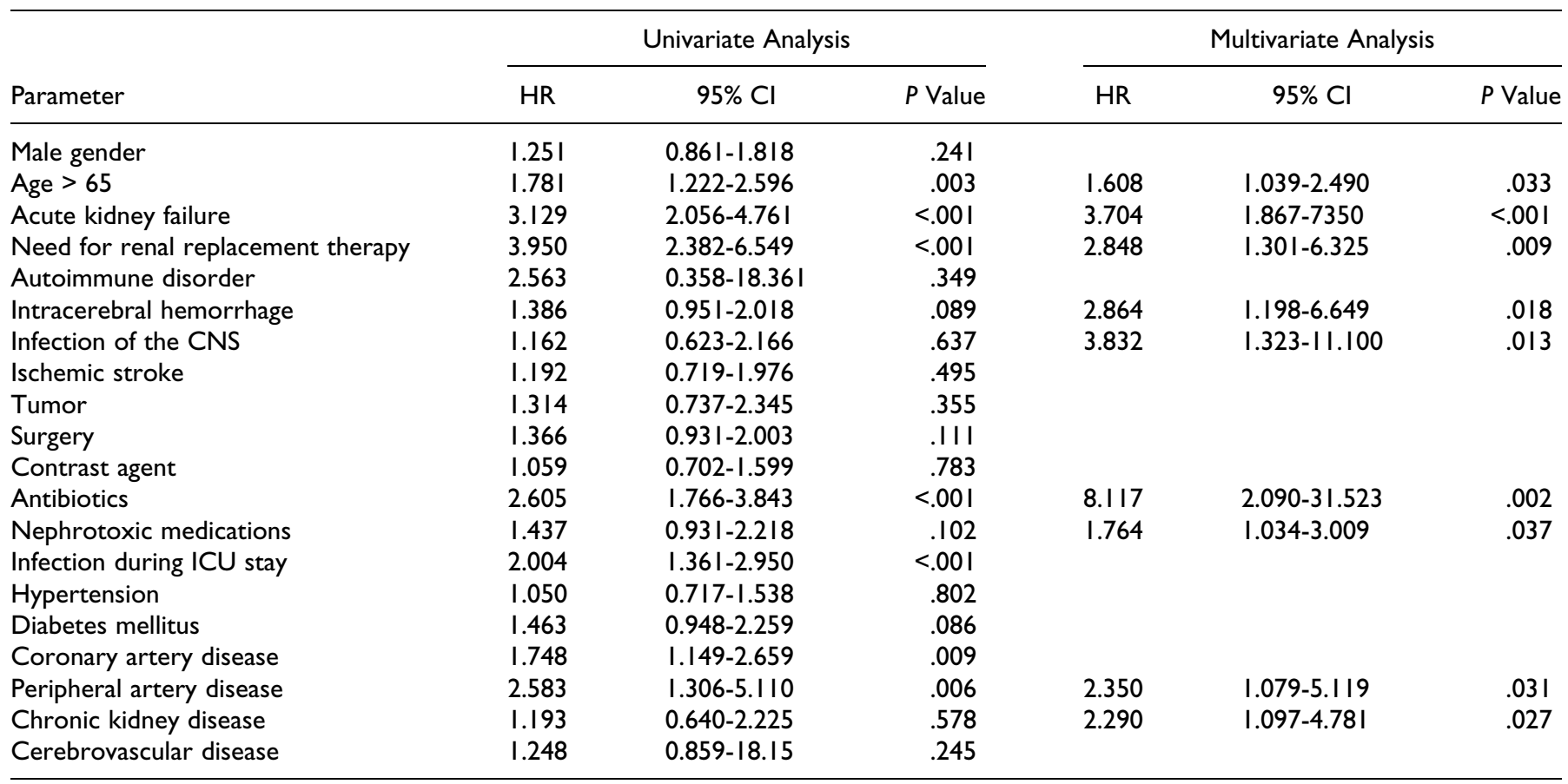

Abbreviations: $\mathrm{Cl}$, confidence interval; CNS, central nervous system; HR, hazard ratio; ICU, intensive care unit.

${ }^{a}$ All $P$ values reported are 2 -sided, and all confidence intervals are $95 \%$ intervals. Statistical significance was defined as $P \leq .05$.

patients with subarachnoid hemorrhage undergoing at least 1 contrast study of $4 \% .{ }^{34}$ In patients with acute stroke undergoing emergency computed tomography angiography, incidence of CI-AKI was $3 \%$, though the authors concluded that contrast agents could be used even in the absence of a creatinine result to minimize treatment delays. ${ }^{35}$

We are aware of the limitations of our study. First, the retrospective character and collection of data only in a single center may limit the transferability of our results to other settings. Furthermore, as we looked at a general population drawing conclusions for individual cohorts can be difficult. Nonetheless, we provide broad epidemiologic data on AKI in neurocritical care. Second, the use of a single serum creatinine value prior to admission or the estimation of kidney function on basis of a serum creatinine value at admission may have led to underestimation of preexisting CKD. On the other hand, kidney function might have been acutely reduced at the time of admission leading to an underestimation of the true incidence of AKI in our population. Additionally, the exclusion of patients with "allow natural death" or withdrawal of care orders leads to the exclusion of patients who may have had AKI. Moreover, patients where dialysis was indicated but declined by family or the patient himself were not specifically analyzed. Third, only patients with severe AKI (AKIN-stage $\geq 2$ ) were analyzed. Slight changes in serum creatinine, which are associated with unfavorable outcome, were not rated in this study. However, we could clearly show that impact of AKI increases with progression through AKIN stages with the greatest impact on survival if dialysis is needed.

In conclusion, AKI is common and is associated with a prolonged ICU stay, a longer duration of mechanical ventilation, and an increased mortality among neurocritically ill patients. In our cohort of neurocritically ill patients, main cause for AKI was sepsis. Therefore, further investigations are warranted to proof if careful prevention and treatment of infectious complications lower the incidence of AKI and hereby improve outcome in neurocritical care.

\section{Acknowledgments}

The authors gratefully acknowledge the work of the staff of the neurocritical care unit and the staff of the dialysis unit.

\section{Declaration of Conflicting Interests}

The author(s) declared no potential conflicts of interest with respect to the research, authorship, and/or publication of this article.

\section{Funding}

The author(s) received no financial support for the research, authorship, and/or publication of this article.

\section{References}

1. Zygun D. Non-neurological organ dysfunction in neurocritical care: impact on outcome and etiological considerations. Curr Opin Crit Care. 2005;11(2):139-143.

2. Hoste EA, Clermont G, Kersten A, et al. RIFLE criteria for acute kidney injury are associated with hospital mortality in critically ill patients: a cohort analysis. Crit Care. 2006;10(3):R73.

3. Hsu RK, McCulloch CE, Dudley RA, Lo LJ, Hsu CY. Temporal changes in incidence of dialysis-requiring AKI. J Am Soc Nephrol. 2013;24(1):37-42. 
4. Uchino S, Kellum JA, Bellomo R, et al; Beginning and Ending Supportive Therapy for the Kidney (BEST Kidney) Investigators. Acute renal failure in critically ill patients: a multinational, multicenter study. JAMA. 2005;294(7):813-818.

5. Case J, Khan S, Khalid R, Khan A. Epidemiology of acute kidney injury in the intensive care unit. Crit Care Res Pract. 2013;2013: 479730.

6. Ostermann M, Chang RWS. Acute kidney injury in the intensive care unit according to RIFLE. Crit Care Med. 2007;35(8): 1837-1843.

7. Metnitz PG, Krenn CG, Steltzer H, et al. Effect of acute renal failure requiring renal replacement therapy on outcome in critically ill patients. Crit Care Med. 2002;30(9):2051-2058.

8. Piccinni P, Cruz DN, Gramaticopolo S, et al; NEFROINT Investigators. Prospective multicenter study on epidemiology of acute kidney injury in the ICU: a critical care nephrology Italian collaborative effort (NEFROINT). Minerva Anestesiol. 2011;77(11): 1072-1083.

9. Chertow GM, Burdick E, Honour M, Bonventre JV, Bates DW. Acute kidney injury, mortality, length of stay, and costs in hospitalized patients. J Am Soc Nephrol. 2005;16(11):3365-3370.

10. Barrantes F, Tian J, Vazquez R, Amoateng-Adjepong Y, Manthous CA. Acute kidney injury criteria predict outcomes of critically ill patients. Crit Care Med. 2008;36(5):1397-1403.

11. Lo LJ, Go AS, Chertow GM, et al. Dialysis-requiring acute renal failure increases the risk of progressive chronic kidney disease. Kidney Int. 2009;76(8):893-899.

12. Coca SG, Singanamala S, Parikh CR. Chronic kidney disease after acute kidney injury: a systematic review and meta-analysis. Kidney Int. 2012;81(5):442-448.

13. Covic A, Schiller A, Mardare NG, et al. The impact of acute kidney injury on short-term survival in an Eastern European population with stroke. Nephrol Dial Transplant. 2008;23(7): 2228-2234.

14. Tsagalis G, Akrivos T, Alevizaki M, et al. Renal dysfunction in acute stroke: an independent predictor of long-term all combined vascular events and overall mortality. Nephrol Dial Transplant. 2009;24(1):194-200.

15. Yahalom G, Schwartz R, Schwammenthal Y, et al. Chronic kidney disease and clinical outcome in patients with acute stroke. Stroke. 2009;40(4):1296-1303.

16. Li N, Zhao WG, Zhang WF. Acute kidney injury in patients with severe traumatic brain injury: implementation of the acute kidney injury network stage system. Neurocrit Care. 2011;14(3): 377-381.

17. Corral L, Javierre CF, Ventura JL, Marcos P, Herrero JI, Mañez R. Impact of non-neurological complications in severe traumatic brain injury outcome. Crit Care. 2012;16(2):R44.

18. Molshatzki N, Orion D, Tsabari R, et al. Chronic kidney disease in patients with acute intracerebral hemorrhage: association with large hematoma volume and poor outcome. Cerebrovasc Dis. 2011;31(3):271-277.

19. Zacharia BE, Ducruet AF, Hickman ZL, et al. Renal dysfunction as an independent predictor of outcome after aneurysmal subarachnoid hemorrhage: a single-center cohort study. Stroke. 2009; 40(7):2375-2381.
20. Le Gall JR, Lemeshow S, Saulnier F. A new Simplified Acute Physiology Score (SAPS II) based on a European/North American multicenter study. JAMA. 1993;270(24):2957-2963.

21. Mehta RL, Kellum JA, Shah SV, et al; Acute Kidney Injury Network. Acute Kidney Injury Network: report of an initiative to improve outcomes in acute kidney injury. Crit Care. 2007;11(2):R31.

22. Bone RC, Balk RA, Cerra FB, et al. Definitions for sepsis and organ failure and guidelines for the use of innovative therapies in sepsis. The ACCP/SCCM Consensus Conference Committee. American College of Chest Physicians/Society of Critical Care Medicine. Chest. 1992;101(6):1644-1655.

23. American Thoracic Society; Infectious Diseases Society of America. Guidelines for the management of adults with hospitalacquired, ventilator-associated, and healthcare-associated pneumonia. Am J Respir Crit Care Med. 2005;171(4):388-416.

24. Chua HC, Lew TW, Ng PY, Yeo TT. Advances in neurointensive care. Ann Acad Med Singapore. 2001;30(3):300-309.

25. Rincon F, Mayer SA. Neurocritical care: a distinct discipline? Curr Opin Crit Care. 2007;13(2):115-121.

26. Zygun DA, Doig CJ, Gupta AK, et al. Non-neurological organ dysfunction in neurocritical care. J Crit Care. 2003;18(4): 238-244.

27. Moore EM, Bellomo R, Nichol A, Harley N, Macisaac C, Cooper DJ. The incidence of acute kidney injury in patients with traumatic brain injury. Ren Fail. 2010;32(9):1060-1065.

28. Odutayo A, Adhikari NK, Barton J, et al. Epidemiology of acute kidney injury in Canadian critical care units: a prospective cohort study. Can J Anaesth. 2012;59(10):934-942.

29. Thakar CV, Christianson A, Freyberg R, Almenoff P, Render ML. Incidence and outcomes of acute kidney injury in intensive care units: a Veterans Administration study. Crit Care Med. 2009; 37(9):2552-2558.

30. Nisula S, Kaukonen KM, Vaara ST, et al. Incidence, risk factors and 90-day mortality of patients with acute kidney injury in Finnish intensive care units: the FINNAKI study. Intensive Care Med. 2013;39(3):420-428.

31. Bagshaw SM, Uchino S, Bellomo R, et al; Beginning and Ending Supportive Therapy for the Kidney (BEST Kidney) Investigators. Septic acute kidney injury in critically ill patients: clinical characteristics and outcomes. Clin J Am Soc Nephrol. 2007;2(3): 431-439.

32. Plataki M, Kashani K, Cabello-Garza J, et al. Predictors of acute kidney injury in septic shock patients: an observational cohort study. Clin J Am Soc Nephrol. 2011;6(7):1744-1751.

33. Gursel G, Demir N. Incidence and risk factors for the development of acute renal failure in patients with ventilator-associated pneumonia. Nephrology (Carlton). 2006;11(3):159-164.

34. Ray B, Rickert KL, Welch BG, et al. Development of contrastinduced nephropathy in subarachnoid hemorrhage: a single center perspective. Neurocrit Care. 2013;19(2):150-156.

35. Krol AL, Dzialowski I, Roy J, et al. Incidence of radiocontrast nephropathy in patients undergoing acute stroke computed tomography angiography. Stroke. 2007;38(8):2364-2366.

36. Mostofsky E, Wellenius GA, Noheria A, et al. Renal function predicts survival in patients with acute ischemic stroke. Cerebrovasc Dis. 2009;28(1):88-94. 